\title{
Estudio piloto comportamental de Panthera onca
}

\author{
Behavioral pilot study in Panthera onca
}

Fecha recepción: 03 de marzo de 2014

Fecha Aprobación: 15 de junio de 2014
Ginette Isabel Chavarro-Tucán', Adriana Patricia Rojas-Rodríguez ${ }^{2}$

\section{Resumen}

En el presente artículo se determinó la respuesta comportamental a un plan de enriquecimiento ambiental en jaguares, y las variaciones en los niveles de metabolitos de glucocorticoides. Se observó el comportamiento de dos hembras en el Bioparque Los Ocarros, a través de monitoreos de una hora tres veces al día, dos días a la semana, durante cuatro semanas, para identificar la diferencia comportamental de cada individuo frente a un plan de enriquecimiento y la ausencia de este. Adicionalmente, se tomaron muestras de materia fecal los días siguientes al monitoreo, las cuales fueron mantenidas a $-20{ }^{\circ} \mathrm{C}$ y posteriormente analizadas con un kit de cortisol para suero y plasma de $\mathrm{DRG}^{\circledR}$, mediante la técnica ELISA. Se observó que el enriquecimiento resultó ser biológicamente significativo para los ejemplares, debidoa queaumentó el número decomportamientos naturales, su frecuencia de presentación y disminuyó la presentación de estereotipias para el caso de uno de los ejemplares. No se observó una variación significativa en los niveles de metabolitos de glucocorticoides. El enriquecimiento aumenta la actividad en los individuos; al no encontrarse una variación en los niveles de metabolitos de glucocorticoides se sugiere, para un próximo estudio, aumentar el tiempo de muestreo.

Palabras clave: Estrés fisiológico, Bienestar animal, Inmunoadsorción enzimática.

\begin{abstract}
This paper determinate the behavioral responses to an environmental enrichment plan in jaguars, and the variations in the levels of glucocorticoid metabolites. We monitored the behavior of two jaguar females in the Bioparque Los Ocarros one hour three times per day, two days per week during a total of four weeks. The goal was to identify behavioral differences in the jaguars with and without the enrichment plan. In addition, we took fecal samples the days after monitoring and stored them at a $-20{ }^{\circ} \mathrm{C}$ until their analysis with ELISA test. The enrichment increased de natural behavior in number and frequency and decrease the presentation of stereotypes for one of the females. No significant variation in the levels of glucocorticoid metabolites was observed. Enrichment increases the activity in individuals, but a variation in the levels of glucocorticoid metabolites was not found. We suggest to increase the sampling time in future studies.
\end{abstract}

Keywords: Physiological Stress, Animal Welfare, Enzyme-linked Immunosorbent Assay. 


\section{Introducción}

La disminución del hábitat de la fauna silvestre, su caza furtiva, su consumo indiscriminado y su comercio ilegal, entre otras, son problemáticas que llevan al aumento de animales en cautiverio. Cuando los ejemplares que se encuentran cautivos no reúnen las condiciones para ser liberados, deben permanecer bajo condiciones que les brinden bienestar y les garanticen un estado de salud favorable y, por consiguiente, su conservación. Sin embargo, a pesar de los esfuerzos, las condiciones en que vive la fauna en cautiverio limitan la expresión del comportamiento natural y pueden llevar a estrés crónico en los ejemplares, y, con esto, a la predisposición a un sinnúmero de enfermedades (1). Los felinos en cautiverio, por ejemplo, presentan múltiples comportamientos agonistas, debido a las condiciones limitadas $(2,3,4)$.

El programa de enriquecimiento ambiental es una herramienta que busca mitigar estos impactos negativos a partir del cambio, día tras día, de las condiciones del encierro $(5,6)$. En el caso particular de los jaguares (Panthera onca) se reportan estudios de enriquecimiento en el Parque zoológico "Yaguar xoo", en Oaxaca, México (2); en el Jardín Zoológico de Buenos Aires, Argentina (3), y en la UMA "Los deseos", en México (7).

Paralelo a estas técnicas de enriquecimiento en felinos y demás animales silvestres surgen métodos que buscan evaluar la efectividad de estas, como lo son: el análisis comportamental y la medición de metabolitos de cortisol en heces $(6,8,9)$, y aunque en los estudios realizados no se encuentra un patrón claro en la disminución de los niveles de cortisol durante el enriquecimiento, en muchos de los casos sí se observa $(2,5,7,8)$.

El presente trabajo buscó identificar las variaciones en el comportamiento y en los niveles de metabolitos de cortisol en heces en dos ejemplares de Panthera onca sometidos a un plan de enriquecimiento.

\section{Materiales y Métodos}

Investigación básica realizada en el Bioparque Los Ocarros, ubicado en la vereda Vanguardia alta, del municipio de Villavicencio, departamento del Meta; con coordenadas $4^{\circ} 11^{\prime}$ 9, $37^{\prime \prime}$ Norte y $73^{\circ} 36^{\prime} 32,85^{\prime \prime}$ Oeste (10), y una altitud de 417 m.s.n.m.; el parque tiene una extensión de 5,2 hectáreas, de las cuales 1,2 son espejo de agua, y registra precipitaciones promedio anuales de $4.300 \mathrm{~mm}$ y una temperatura promedio de 27 ${ }^{\circ} \mathrm{C}$ (10). Los animales estudiados fueron dos ejemplares hembras, una en estado de desarrollo biológico juvenil, y la otra, adulta. Se realizó una fase de campo, durante la cual se realizaron los monitoreos, la recolección de heces y su almacenamiento; enseguida se realizó una fase de laboratorio en la cual se llevó a cabo el análisis comportamental y la medición de los metabolitos de glucocorticoides. Finalmente, se analizaron los resultados. Los datos sobre el comportamiento fueron recolectados los días martes y jueves, tres veces cada día durante una hora a intervalos de cinco minutos por ejemplar, a través de la metodología de muestreo exploratorio (11).

Plan de enriquecimiento. Para la elaboración y ejecución del plan de enriquecimiento se tuvo en cuenta la historia natural del jaguar y la disponibilidad de espacio, tiempo, materiales y seguridad del procedimiento. Los enriquecimientos fueron de tipo trófico, sensorial, ocupacional y de ambientación, los cuales se implementaron independientemente de que los ejemplares se encontraran en área de exhibición o área de manejo.

Muestras. Se tomó muestra de material fecal completa por cada individuo en las horas de la mañana, los días miércoles y viernes, correspondientes al día siguiente del monitoreo, con y sin enriquecimiento, momento que coincide con el pico de excreción de metabolitos de cortisol $(12,13)$; se homogeneizó y se almacenó dentro de un recipiente plástico (para muestra de orina), debidamente rotulado, a una temperatura de $-20^{\circ} \mathrm{C}$, hasta el momento de ser procesada (6, $14,15,16)$.

Extracción de los metabolitos de glucocorticoides. La extracción se realizó usando el método de Vortex $(2,8)$ :

1. Se descongelaron las muestras a temperatura ambiente.

2. Se tomó $1 \mathrm{~g}$ de materia fecal, se disolvió en 5 $\mathrm{ml}$ de etanol acuoso (90\%) y se calentó al baño María durante 20 minutos.

3. Se homogeneizó la muestra y se centrifugó a 1200 gravedades (g) durante 15 minutos. 
4. Se recuperó el sobrenadante, con ayuda de una micropipeta (4 $\mathrm{ml}$ apróx.).

5. Se resuspendió el pellet en $5 \mathrm{ml}$ de etanol acuoso $(90 \%)$.

6. Se mezcló utilizando vortex durante 1 minuto.

7. Se centrifugó a $1200 \mathrm{~g}$ durante 15 minutos.

8. Se recuperó el sobrenadante con ayuda de una micropipeta (4 $\mathrm{ml}$ apróx.).

9. Se combinaron ambos sobrenadantes $(8 \mathrm{ml}$ apróx.) y se secó completamente con ayuda de un rotovapor a $40{ }^{\circ} \mathrm{C}$. El procedimiento tardó alrededor de 5 minutos por cada muestra.

10. Se redisolvió la muestra en $1 \mathrm{ml}$ de metanol.

11. Se diluyó 1:10 en una solución buffer fosfato (PBS) $\quad(0.01 \mathrm{M} \mathrm{NaPO} 4 ; 0.14 \mathrm{M} \mathrm{NaCl} ; 0.5 \%$ Albumina sérica bovina; $\mathrm{pH}$ 7.4). El PBS reportado en el protocolo contenía Azida sódica; para este caso no se utilizó, debido a que el Kit DRG ${ }^{\circledR}$ especifica que las muestras no deben contenerla.

El volumen utilizado fue de $40 \mu \mathrm{L}$ de la muestra en $360 \mu \mathrm{L}$ de PBS. De este volumen final se tomó finalmente la muestra que fue procesada mediante la técnica ELISA (6).

\section{Resultados}

Se monitoreo durante un total de 42 horas, y para el caso de la ejemplar Luna se observaron diferencias biológicas entre las semanas sin enriquecimiento (semana 2 y 4) y las semanas con enriquecimiento (semanas 1 y 3 ), donde los periodos de descanso disminuyeron (Tabla I).

Tabla I. Frecuencia de presentación de las unidades comportamentales en cada semana para la ejemplar Luna.

\begin{tabular}{ccccccccc}
\hline $\begin{array}{c}\text { Unidad } \\
\text { Semana }\end{array}$ & Caminar & Olfatear & Saltar & Jugar & Lamerse & Pacing & Dormir & Descansar \\
\hline 2 & 7 & 1 & 0 & 0 & 6 & 8 & 9 & 11 \\
3 & 28 & 22 & 3 & 1 & 5 & 4 & 5 & 3 \\
4 & 12 & 0 & 1 & 0 & 13 & 6 & 0 & 12 \\
5 & 25 & 11 & 9 & 3 & 6 & 1 & 1 & 7 \\
\hline
\end{tabular}

Para el caso de la ejemplar Mariposa también se observaron diferencias biológicas entre las semanas (Tabla II).

Tabla II. Frecuencia de presentación de las unidades comportamentales en cada semana para la ejemplar Mariposa.

\begin{tabular}{ccccccccc}
\hline $\begin{array}{c}\text { Unidad } \\
\text { Semana }\end{array}$ & Caminar & Olfatear & Saltar & Jugar & Lamerse & Pacing & Dormir & Descansar \\
\hline 2 & 10 & 3 & 6 & 11 & 2 & 0 & 8 & 3 \\
3 & 44 & 19 & 5 & 18 & 5 & 1 & 2 & 6 \\
4 & 16 & 2 & 5 & 1 & 11 & 0 & 4 & 11 \\
5 & 33 & 12 & 12 & 18 & 6 & 0 & 2 & 0 \\
\hline
\end{tabular}

Niveles de metabolitos de glucocorticoides. Se realizó la prueba ELISA, y para el caso de la ejemplar Luna se encontró que los niveles permanecieron similares, exceptuando la semana 5 con enriquecimiento, en la cual se observó un aumento de estos. Para el caso de la ejemplar Mariposa, los niveles permanecieron bajos, si bien se observó un aumento de estos para finales de la cuarta semana y para la quinta semana (Tabla III). 
Tabla III. Variaciones en los niveles de metabolitos de glucocorticoides en las ejemplares Luna y Mariposa.

\begin{tabular}{cccc}
\hline SEMANA & DíA & Niveles de metabolitos de glucocorticoides $(\mathbf{n g} / \mathbf{m l})$ \\
\hline 2 & & Luna & Mariposa \\
& Martes & 52 & 14 \\
& Jueves & 18 & 49 \\
3 & Martes & 54 & 12 \\
& Jueves & 16 & 12 \\
& Martes & 16 & 15 \\
4 & Jueves & 16 & 40 \\
& & & 45 \\
& Martes & 194 & 41 \\
& Jueves & 661 & \\
\hline
\end{tabular}

\section{Discusión}

Los valores encontrados en el estudio no fueron estadísticamente significativos para probar la hipótesis de que el enriquecimiento ambiental es efectivo para mejorar el bienestar de los animales que son mantenidos en cautiverio, contrario a otros estudios $(7,17,18,19)$; posiblemente, esto se debió al corto tiempo de muestreo (no hubo un tiempo de adaptación de los ejemplares a los enriquecimientos) y al número limitado de muestras, que no permitió observar el comportamiento a largo plazo de las fluctuaciones en los niveles de cortisol; sin embargo, sí fue posible observar que existen diferencias biológicas entre la implementación o no de enriquecimiento y la actividad de los individuos.

De esta manera, se observó que los ejemplares sometidos al plan de enriquecimiento aumentaron su actividad comportamental, caminando durante una mayor cantidad de veces y de tiempo, y registrando comportamientos como olfatear y explorar, que no fueron observados durante los días sin enriquecimiento.

Desde la perspectiva del observador, los enriquecimientos implementados permitieron a los ejemplares realizar un mayor número de comportamientos durante una determinada unidad de tiempo; mientras que en el ambiente adinámico, sin la presencia de estímulos constantes, realizaron el mismo recorrido durante el día y permanecieron descansando la mayor parte del tiempo. En el ambiente idéntico, pero esta vez dinámico, con la presencia de elementos nuevos y estímulos constantes, realizaron un mayor número de actividades durante la misma unidad de tiempo, dedicando más a la exploración y la búsqueda trófica, disminuyendo los tiempos de descanso y quietud $(2,20)$.

En cuanto a la presentación de estereotipias, se observó que en el ejemplar Luna la estereotipia pacing se vio notablemente disminuida, pasando en el primer muestreo de ocho observaciones a cuatro, y de seis a uno en el segundo, durante el enriquecimiento.

Algunos estudios señalan que el aumento de las estereotipias contribuye a la disminución de estrés en los individuos en cautiverio $(18,20)$, pero otros encontraron lo contrario: que al disminuir las estereotipias disminuyeron los niveles fisiológicos de estrés (21). Para este estudio, el primer monitoreo de enriquecimiento muestra una disminución de la estereotipia, pero una permanencia en los niveles de metabolitos de glucocorticoides, y para el segundo monitoreo, además de observar una disminución en la presentación de la estereotipia, se observó un notable aumento en los niveles de metabolitos de glucocorticoides. Al no existir un resultado similar para los dos casos será necesario aumentar el número de muestreos, con el fin de 
registrar la tendencia que estos tienen a largo plazo, además de aumentar el número de enriquecedores y sus variaciones en la presentación y considerar las condiciones climáticas, que posiblemente sean un factor de estrés para los ejemplares.

Una de las posibles razones por las cuales Luna presenta la estereotipia pacing es su mayor tamaño y su mayor estado de desarrollo biológico que Mariposa, y a que ya tiene madurez sexual. Los rangos de acción en vida silvestre para animales adultos son más grandes que los de los ejemplares con un estado de desarrollo biológico menor, y el pequeño tamaño de su hábitat no les permite realizar el mismo número de actividades que realizarían en vida silvestre $(21,22)$. Posiblemente, si las condiciones se mantienen para los dos ejemplares, Mariposa podría comenzar a presentar estereotipias a medida que su estado de desarrollo biológico avance.

Otra de las posibles razones por las cuales Luna presenta la estereotipia pacing es su temperamento, que puede influir en la aparición de estereotipias, dado que la manera como el ejemplar reacciona a su entorno determina la respuesta a desencadenar $(21,23)$. Para el caso de Mariposa, el ejemplar más joven, se observa mayor disposición al juego y a la interacción con el público, el hábitat y el cuidador. Por el contrario, Luna demuestra poca empatía hacia el público, hace menor uso de los elementos del hábitat, dedica más tiempo a descansar y muchas veces demuestra comportamientos agresivos hacia el cuidador.

Para la medición de los metabolitos de glucocorticoides se tuvieron en cuenta las fluctuaciones en los niveles relativos de cortisol para cada individuo $(24,25,26,27)$. Para este caso, se observó un comportamiento similar de los resultados en ambos ejemplares para el caso de Luna; para la primera semana de enriquecimiento los valores permanecieron muy similares a los de la primera semana sin enriquecimiento, mientras que para la segunda semana de enriquecimiento estos aumentaron de $16 \mathrm{ng} / \mathrm{ml}$ (sin enriquecimiento) a $661 \mathrm{ng} / \mathrm{ml}$. En el ejemplar Mariposa ocurrió el mismo comportamiento, aunque el aumento para la segunda semana fue tan solo de $15 \mathrm{ng} / \mathrm{ml}$ ( $\sin$ enriquecimiento) a $41 \mathrm{ng} / \mathrm{ml}$ con enriquecimiento.

¿Qué ocurrió en la segunda semana de enriquecimiento que pudiera llevar al aumento de los niveles en ambos ejemplares? Para el caso de la primera semana no se utilizaron enriquecedores tróficos (ración colgada o escondida), la segunda semana de enriquecimiento ambos días tuvieron un componente trófico. Este desafío de obtención del alimento pudo llevar al aumento de los niveles de cortisol para ambos ejemplares.

Es posible señalar que los enriquecimientos que se aplican diferentes al trófico se pueden interpretar como una novedad para los individuos y como una alternativa de la ocupación del tiempo que estos pueden elegir o no. Mientras que la falta de ración o esconderla, suministrarla a horarios diferentes o ponerla en lugares de difícil acceso y diferentes a los que el ejemplar está acostumbrado pueden constituir un desafío que provoca una activación del eje hipotálamo-hipofisiario-adrenal, debido a que este enriquecimiento es claramente un agente estresante para la rutina y el reloj biológico del ejemplar.

Para el caso de Luna, la última muestra arrojó un valor de $661 \mathrm{ng} / \mathrm{ml}$ (la más alta registrada para todas las muestras); al no tener muestras subsecuentes no es posible conocer si este valor se mantuvo o si, por el contrario, correspondió a una fluctuación normal por la aplicación del enriquecimiento, o si se debió a un factor diferente no tenido en cuenta en este estudio, como pudo ser el ayuno del día anterior.

La cantidad de cortisol que la ración pudiera tener también pudo verse reflejada en el aumento de los niveles en ambos ejemplares; sin embargo, es importante destacar que la ración suministrada no fue diferente durante las cuatro semanas, lo que cambio fue la forma como se suministró durante la segunda semana de enriquecimiento.

El flujo de visitantes también pudo contribuir a aumentar los niveles de estrés; debido a la naturaleza solitaria y salvaje de estos individuos, es posible afirmar que la proximidad con los seres humanos es un factor de estrés, ya que estos no se encuentran habituados a interactuar con ellos (24, $25,26)$.

Finalmente, los programas de bienestar animal que se implementan en los zoológicos no tienen un fin diferente al de mejorar las condiciones de vida de los ejemplares que se encuentran en cautiverio. Un estudio reciente (1) demuestra que no basta 
con conocer la historia natural de determinada especie, sino que es importante estudiar de cerca los individuos para así elaborar programas de enriquecimiento que se acerquen más a las necesidades de los ejemplares y contribuyan con el bienestar de un ejemplar en particular.

Para el caso de este estudio fue posible establecer que para la ejemplar Mariposa se deben crear planes de enriquecimiento encaminados a un mayor uso del espacio; la colocación de troncos y ramas, de elementos que sirvan de juguetes y de otros que contribuyan a un aumento de la actividad es útil a la hora de suplir las necesidades de este ejemplar juvenil. Para el caso de Luna se debe buscar brindar espacios donde el ejemplar pueda refugiarse, y planear horarios en los cuales se asegure que los días en los que sale a exhibición sean también los de menor flujo de visitantes.

Para ambos ejemplares, brindar un mayor número de presas vivas y crear días en el zoológico durante los cuales, una vez cerrado el parque, se realice una hora de enriquecimiento en exhibición con presas vivas, y así los ejemplares pueden desplegar un mayor número de comportamientos naturales en un espacio más amplio; siempre procurando variar los enriquecedores, su periodicidad y forma de suministro, para así evitar el acostumbramiento de los ejemplares.

\section{Conclusiones}

Los niveles de metabolitos de glucocorticoides no presentaron un comportamiento homogéneo, y se observó tanto una disminución como un aumento de estos en condiciones de enriquecimiento para los dos ejemplares; para el caso del segundo muestreo se observó un aumento, siendo más significativo para el caso de Luna.

Se sugiere para futuros estudios un mayor número de muestras por ejemplar, con el fin de observar el comportamiento que presentan los niveles de cortisol y las variaciones en el comportamiento a lo largo de un periodo mayor.

\section{Referencias}

(1) Whitham J, Wielebnowski N. New directions for zoo animal welfare science. Appl. Anim. Behav. Sci 2013; 147: 247-260.
(2) Castillo C, Unda K, Lara C, SerioJ. Enriquecimiento ambiental y su efecto en la exhibición de comportamientos estereotipados en jaguares (Panthera onca) del Parque zoológico "Yaguar Xoo", Oaxaca. Acta Zool. Méx 2012; 28(2): 365377.

(3) Merélle X. (2010). Enriquecimiento ambiental en Jaguareté (Panthera onca): una herramienta fundamental para su bienestar en cautiverio. Reportes Tigreros, 3: 1-13.

(4) Rangel A, Alfaro J, Valdez R, Romano M, Serio J. Stress in Yucatan spider monkeys: effects of environmental conditions on fecal cortisol levels in wild and captive populations. Anim. Conserv 2009; 12: 496-502.

(5) Burgener N, Gusset M. Frustrated appetitive foraging behavior, stereotypic pacing, and fecal glucocorticoid levels in Snow Leopards (Uncia uncia) in the Zurich Zoo. J Appl Anim Welf Sci 2008; 11: 74-83.

(6) Rodríguez C, Arias L. Validación y aplicación de la prueba ELISA para medir cortisol fecal en jaguar (Panthera onca) y puma (Puma concolor) durante un programa de enriquecimiento ambiental en el zoológico Jaime Duque. Rev Med Vet 2005; (10): 53-64.

(7) Goméz M. Enriquecimiento ambiental para un oso negro hembra (Ursus americanus) y un jaguar macho (Panthera onca) en la UMA rancho "Los deseos", en el municipio de Banderilla, Veracruz. Trabajo de grado, Facultad de Biología; Universidad Veracruzana 2010.

(8) Novak M, Hamel A, Kelly B, Dettmer A, Meyer J. Stress, the HPA axis, and nonhuman primate well-being: A review. Appl. Anim. Behav. Sci 2013; 143: 135-149.

(9) Conforti V, Morato R, Augusto M, De Oliveira E, Sousa, L, De Avila D et al. Non invasive monitoring of adrenocortical function in captive jaguars (Panthera onca). Zoo Biol 2011; 30: 1-16.

(10)Villavicencio. Plan de Ordenamiento Territorial Villavicencio (en línea) 2014 (fecha de acceso 28 de marzo de 2015).

(11)Zerda E. Comportamiento Animal: Introducción, Métodos y Prácticas. Bogotá: Unilibros, Universidad Nacional de Colombia 2004.

(12)Hämäläinen A, Heistermann M, Fenosoa Z, Kraus C. Capture stress in wild gray mouse lemurs via repeated fecal sampling: Method validation and the influence of prior experience and handling protocols on stress responses. Gen Comp Endocr 2014; 195: 68-79.

(13) Young K, Walker S, Lanthier C, Waddell W, Monfort S, Browna J. Noninvasive monitoring of adrenocortical activity in carnivores by fecal 
glucocorticoid analyses. Gen Comp Endocr 2004; 137: 148-165.

(14) Narayan EJ, Clark G, Martin P, Parnell T, Mucci $\mathrm{Al}$, Hero JM. Faecal cortisol metabolites in Bengal (Panthera tigris tigris) and Sumatrantigers (Panthera tigris sumatrae). Gen Comp Endocr 2013; 194: 318-325.

(15)Palme R, Touma C, Arias N, Dominchin M, Lepschy F. Steroidextraction: Getthebestout of faecal samples. Vet Med Austria 2013; 100: 238246.

(16)Antunes E, Nichi M, Guimarães M. Comparison of two commercial kits and $\mathrm{t}$ wo extraction methods for fecal glucocorticoid analysis in ocelots (Leopardus pardalis) submitted to ACTH challenge. Pesquisa Vet Brasil 2008; 28 (7): 329334.

(17)Eguizábal G, Palme R, Villarreal D, Dal Borgo C, DI Rienzo J, Busso J. Assessment of adrenocortical activity and behavior of the collared anteater (Tamandua tetradactyla) in response to foodbased environmental enrichment. Rev. Zoo Biol (en línea) 2013 (fecha de acceso 4 de diciembre de 2014). Disponible en: www.scopus.com.

(18)Vivas A, Jiménez G, Rodríguez C. Efecto del enriquecimiento ambiental en la respuesta fisiológica y comportamental de osos de anteojos en un zoológico de Colombia. Memorias de la conferencia interna en medicina $y$ aprovechamiento de fauna silvestre, exótica y no convencional 2012; 8(2): 10-15.

(19)Rangel A, Alfaro J, Valdez R, Romano M, Serio J. Stress in Yucatan spider monkeys: effects of environmental conditions on fecal cortisol levels in wild and captive populations. Anim. Conserv 2009; 12: 496-502.

(20)Zaragoza F, Ibáñez M, Mas B, Laiglesia S, Anzola $B$. Influence of environmental enrichment in captive chimpanzees (Pan troglodytes spp.) and gorillas (Gorilla gorilla gorilla): Behavior and faecal cortisol levels. Rev. Fac. Agron 2012; 21(5): 447-456.

(21)Shepherdson, D; Lewis, K; Carlstead, K; Baumanc, J. Individual and environmental factors associated with stereotypic behavior and fecal glucocorticoid metabolite levels in zoo housed polar bears. Appl. Anim. Behav. Sci 2013; 147: 268-277.

(22)Clubb R y Mason G. Natural behavioural biology as a risk factor in carnivore welfare: How analysing species differences could help zoos improve enclosures. Appl. Anim. Behav. Sci 2007; 102: 303-328.

(23)Ramos D, Reche A, Fragoso L, Palme R, Yanasse N, Gouvêa V, Beck A, Mills D. Are cats (Felis catus) frommulti-cat households more stressed? Evidence from assessment of fecal glucocorticoid metabolite analysis. Physiol. \& Behav 2013; 122: 72-75.

(24)Menargues A, Urios V, Limiñana R. Seasonal pattern of salivary cortisol secretion in the greater one-horned rhino (Rhinoceros unicornis). Anim Welfare 2013; 22(4): 467-472.

(25)Pifarré M, González C, Vázquez C, Romano $M$, Galindo F. The effect of zoo visitors on the behaviour and faecal cortisol of the Mexican wolf (Canis lupus baileyi). Appl. Anim. Behav. Sci, 2012; 136 (1): 57-62.

(26)Rajagopal T, Archunan G y Sekar M. Impact of Zoo Visitors on the Fecal Cortisol Levels and Behavior of an Endangered Species: Indian Blackbuck (Antelope cervicapra) L. J. Appl. Anim. Welfare Sci 2011; 14(1): 18-32.

(27)Morato R, Bueno M, Malmheister P, Verreschi T, Barnabe R. Changes in the fecal concentrations of cortisol and androgen metabolites in captive male jaguars (Panthera onca) in response to stress. Braz J Med Biol Res 2004; 37(12): 19031907. 